\title{
Slow platelet recovery pattern during the 2011 dengue outbreak: a preliminary report
}

\author{
L Gooneratne ${ }^{1}$, M Wimalachandra ${ }^{1}$, J Wijerathna ${ }^{3}$, N Karunathunga $^{3}$, S Jayasinghe ${ }^{2}$
}

\begin{abstract}
During the 2011 dengue outbreak, health care providers in Colombo, Sri Lanka noticed a slow rise in platelet counts in patients recovering from dengue fever when compared to the 2010 outbreak. This study was carried out to confirm this observation. The platelet recovery rates of two groups of patients from 2010 and 2011 ( $n=28$ and $\mathrm{n}=25$ respectively) were computed and compared. The platelet recovery rates, of patients from the 2011 outbreak were found to be slower than the platelet recovery rates of patients from the 2010 outbreak ( $p$ value $=0.0089$ ).
\end{abstract}

Ceylon Medical Journal 2014; 59: 61-63

\section{Introduction}

Dengue fever (DF) and dengue haemorrhagic fever (DHF) is endemic in Sri Lanka. Following the first reported dengue outbreak in 1965, the frequency and magnitude of dengue outbreaks are on the rise. Particularly large outbreaks were reported in the years 2002, 2004 and 2009 $[1,2]$.

Thrombocytopenia in DF recovers with clinical improvement. The increment of the platelet count is used as a feature of recovery as well as a discharge criterion in clinical practice [3]. During the 2011 outbreak, health care providers in Colombo, Sri Lanka observed a slow rise in platelet counts among patients recovering from $\mathrm{DF} / \mathrm{DHF}$ when compared to the 2010 outbreak. This study was carried out to confirm or refute this observation.

\section{Methods}

The Central Hospital is a tertiary care private hospital located in the commercial capital Colombo. Records were retrieved of all patients confirmed with dengue fever who were admitted to the hospital, during the months of June, July and August in 2010 and 2011. Patients between the ages of 12-65 years, without any concurrent conditions were included in the study. They were confirmed to have DF/DHF through clinical assessment by the treating physician and laboratory confirmation by NS1 antigen positivity or positive IgM serology. The protocol for the study was approved by the Ethics Review Committee of the Faculty of Medicine, University of Colombo.

Serial platelet counts performed during the recovery period were extracted from each patient and scatter plots of platelet counts were drawn against time. The above model was converted to a linear curve using the natural logarithm of platelet count of each patient. The Gradient $(\alpha)$ and Intercept $(\ln (\beta))$ of each regression line was recorded and average platelet recovery rate of each patient was calculated using $\alpha$ and $\beta$ as follows.

Platelet recovery rate at time $\mathrm{t}=\frac{d y}{d t}=\alpha \beta \mathrm{e}^{\alpha \mathrm{t}}$

Average platelet recovery rate $=\frac{1}{\mathrm{~T}} \int_{0}^{\mathrm{T}} \alpha \beta e^{\alpha \mathrm{t}} d t=\frac{\beta}{\mathrm{T}}\left[\mathrm{e}^{\alpha \mathrm{t}}-1\right]$

Where $\mathrm{T}$ is the time length in which platelet counts of relevant patients were recorded. The average platelet recovery rates of patients in 2010 were compared with patients in 2011 using the Mann Whitney test. The platelet recovery rates and lowest and highest platelet counts obtained from each patient are shown in Table 1.

\section{Results}

Forty four patients from the year 2010 (Group 1) and 42 patients from the year 2011 (Group 2) fulfilled the inclusion criteria for the study. Depending on the fit of the regression model, we were able to obtain recovery rates for 28 patients in group 1 and 25 patients in group 2 . Therefore 16 patients from group 1 and 17 patients from group 2 had to be excluded from the data analysis as they did not have sufficient data points (less than four data points) to find a reasonable platelet recovery rate. The age $(p=0.67)$ and sex $(p=0.34)$ distribution between the groups did not differ significantly. The results of the Mann Whitney test is given below,

$\mathrm{n}_{1}$-Sample size of smaller group - Platelet recovery rate of $2011=25$

$\mathrm{n}_{2}$ - Sample size of larger group - Platelet recovery rate of $2010=28$

$\mathrm{R}_{1}$ - Sum of the ranks of group with $\mathrm{n}_{1}$ cases $=533$

$R_{2}$ - Sum of the ranks of group with $n_{2}$ cases $=898$

Departments of ${ }^{1}$ Pathology and ${ }^{2}$ Clinical Medicine, Faculty of Medicine, University of Colombo and Department of ${ }^{3}$ Mathematics, Faculty of Science, University of Colombo, Sri Lanka.

Correspondence: LG, e-mail: <viranjan.gooneratne@yahoo.co.uk>. Received 26 September 2012 and revised version accepted 25 November 2013. Competing interests: none declared. 


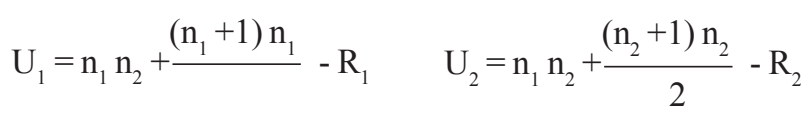

$\mathrm{U}_{1}=492, \mathrm{U}_{2}=208$

Test Statistic $U=U_{2}=\mathbf{2 0 8}\left(U_{2}\right.$ is expected to be smaller, because $R_{2}$ is expected to be larger).

The distribution is approximately normal due to large sample size $(>20)$. Therefore, the Z-value above can be used.

$\mathrm{Z}=\frac{U-\mu_{u}}{\sigma_{u}}$ where $\mu_{\mu} \frac{n_{1} n_{2}}{2}$ and $\sigma_{u}=\sqrt{\frac{n_{1} n_{2}\left(n_{1}+n_{2}+1\right)}{12}}$

$Z=-2.5301$, Corresponding $P$ value $=0.0089$. The platelet recovery rate of 2011 is slower than the platelet recovery rate of 2010 .

\section{Table 1. Platelet recovery rates per patient and lowest and highest platelet counts of each patient, during the 2010 and 2011 dengue outbreaks}

\begin{tabular}{|c|c|c|c|c|}
\hline $\begin{array}{l}\text { Patient } \\
\text { number }\end{array}$ & $\begin{array}{l}\text { Platelet recovery } \\
\text { rates in } 2010 \\
\left(\times 10^{12} / \mathrm{L}\right. \\
\text { per hour })\end{array}$ & $\begin{array}{l}\text { Lowest and } \\
\text { highest platelet } \\
\text { counts in } 2010 \\
\left(\times 10^{12} / \mathrm{L}\right)\end{array}$ & $\begin{array}{c}\text { Platelet } \\
\text { recovery counts } \\
\text { in } 2011 \\
\left(\times 10^{12} / \mathrm{L}\right. \\
\text { per hour })\end{array}$ & $\begin{array}{c}\text { Lowest and } \\
\text { highest } \\
\text { platelet } \\
\text { counts in } \\
2011 \\
\left(\times 10^{12} / \mathrm{L}\right)\end{array}$ \\
\hline 1 & 1.35 & $32-72$ & 0.60 & $17-45$ \\
\hline 2 & 1.61 & $34-117$ & 1.11 & $9-41$ \\
\hline 3 & 1.20 & $15-80$ & 0.44 & $10-52$ \\
\hline 4 & 0.86 & $16-52$ & 0.91 & $18-68$ \\
\hline 5 & 1.67 & $22-62$ & 0.86 & $6-65$ \\
\hline 6 & 0.47 & $20-60$ & 0.63 & $28-60$ \\
\hline 7 & 2.43 & $75-211$ & 0.85 & $9-52$ \\
\hline 8 & 0.86 & $33-98$ & 1.44 & $34-98$ \\
\hline 9 & 1.84 & $10-40$ & 1.06 & $33-80$ \\
\hline 10 & 1.06 & $20-70$ & 0.72 & $10-56$ \\
\hline 11 & 0.88 & $28-85$ & 0.53 & $19-44$ \\
\hline 12 & 1.23 & $21-60$ & 1.06 & $9-62$ \\
\hline 13 & 1.08 & $12-87$ & 0.76 & $18-45$ \\
\hline 14 & 1.42 & $44-127$ & 0.03 & $6-95$ \\
\hline 15 & 1.70 & $10-90$ & 0.48 & $22-59$ \\
\hline 16 & 1.34 & $27-95$ & 0.88 & $18-68$ \\
\hline 17 & 0.58 & $16-65$ & 1.70 & $16-90$ \\
\hline 18 & 1.75 & $24-104$ & 1.81 & $38-138$ \\
\hline 19 & 2.17 & $28-117$ & 1.32 & $40-107$ \\
\hline 20 & 1.31 & $69-126$ & 0.66 & $70-95$ \\
\hline 21 & 1.18 & $24-70$ & 1.56 & $38-155$ \\
\hline 22 & 1.20 & $40-84$ & 2.24 & $13-70$ \\
\hline 23 & 2.46 & $28-161$ & 0.81 & $10-58$ \\
\hline 24 & 1.95 & $30-132$ & 0.34 & $60-83$ \\
\hline 25 & 0.58 & $15-32$ & 2.96 & $25-175$ \\
\hline 26 & 1.74 & $22-83$ & & \\
\hline 27 & 1.02 & $37-115$ & & \\
\hline 28 & 1.43 & $25-152$ & & \\
\hline
\end{tabular}

\section{Discussion}

Through this preliminary study we were able to confirm the validity of the casual observation made by physicians on the slower platelet recovery rates during the 2011 dengue epidemic compared to the 2010 epidemic in the Colombo District.

There was a high number of drop outs in the final analysis due to the lack of data points necessary to fit a suitable regression curve. Therefore we propose that our findings should be confirmed in a prospective study in a larger study sample. However obtaining sufficient platelet counts in patients recovering from dengue will remain a challenge as many patients are discharged and lost to follow up when the platelet counts rise above $50000 / \mu \mathrm{L}$.

In the year 2010 Sri Lanka recorded 34,105 cases of DF and 246 deaths, while in 2011, 28,140 cases were recorded and 185 deaths [4, 5]. During 2010 and 2011 the highest number of cases and the highest number of deaths due to DF/DHF were recorded in the Colombo district. Two peaks in the incidence of dengue cases were observed each year, coinciding with the monsoon rains that swept across the island from May to August (South-West Monsoon) and November to January (North-west monsoon).

There are no published data on viral serotypes or genotypes isolated from Sri Lanka in the years 2010 and 2011. Although there is no systematic surveillance of circulating dengue serotypes in Sri Lanka, several authors have shown the emergence of DEN 1 as the predominant serotype from 2009 [6,7]. This is in contrast to previous studies that showed DEN 2 and DEN 3 as the predominant serotypes until the year $2006[1,2]$. Thus this slow platelet recovery rate could be a result of DEN 1 establishing itself as the predominant circulating serotype in Colombo. It could also be due to different genotypes or clades emerging within the DEN 1 serotype.

The National guidelines on the management of dengue patients require a platelet count of more than 50,000 prior to discharge [3]. This practice was followed in most of the local hospitals despite the strain on resources. Significant delay in the platelet recovery during the 2011 outbreak would have had an impact on the cost of treatment by contributing to both direct (investigations, hospital stay) and indirect (wages lost, travel) costs.

Despite the above shortcomings of small sample size, high rates of missing values and the retrospective nature of the study, these findings show a change in the trend of platelet recovery which has not been reported previously.

\section{References}

1. Messer WB, Vitarana UT, Sivananthan K, et al. Epidemiology of dengue in Sri Lanka before and after the emergence of epidemic dengue hemorrhagic fever. The American Journal of Tropical Medicine and Hygiene 2002; 66: 765-73. 
2. Kanakaratne N, Wahala WMPB, Messer WB, et al. Severe dengue epidemics in Sri Lanka, 2003-2006. Emerging Infectious Diseases 2009; 15: 192.

3. Guidelines for the management of Dengue fever and Dengue haemorrhagic fever in adults. Sri Lanka: Epidemiology Unit, Ministry of Health; 2010.

4. Distribution of Notification (H399) Dengue cases and deaths by months,2010 [Internet] Epidemiology Unit, Ministry of Health, Sri Lanka 2010 [cited 2012 May 8]; Available from: http://www.epid.gov.lk/web/index.php?option=com casesanddeaths $\&$ Itemid $=448 \&$ lang $=$ en .

5. Distribution of Notification (H399). Dengue cases and deaths by month, 2011. [Internet] Epidemiology Unit, Ministry of Health, Sri Lanka 2011 [updated 2012 Feb 10; cited 2012 May 8]; Available from: http://www.epid.gov.lk/web/ index.php?option=com_casesanddeaths\&Itemid $=448 \&$ lang=en.

6. Dissanayake VH, Gunawardena ND, Gunasekara NC, Siriwardhana DR, Senarath N. Shift in the transmission pattern of dengue serotypes and concurrent infection with more than one dengue virus serotype. Ceylon Medical Journal 2011; 56: 176-8.

7. Tissera HA, Ooi EE, Gubler DJ, et al. New dengue virus type 1 genotype in Colombo, Sri Lanka. Emerging Infectious Diseases 2011; 17: 2053-5.

\title{
Methicillin resistant Staphylococcus aureus among nurses in a tertiary care hospital in northern Sri Lanka
}

\author{
U Mahalingam ${ }^{1}, \mathrm{~K}_{\text {Thirunavukarasu }}{ }^{2}, \mathrm{~K}_{\text {Murugananthan }}{ }^{3}$ \\ (Index words: methicillin resistant Staphylococcus aureus (MRSA), infection control, hand hygiene)
}

\begin{abstract}
Methicillin resistant Staphylococcus aureus (MRSA) carriage in the hands among 109 nursing staffs of Teaching Hospital, Jaffna, Sri Lanka was screened. Of those screened, $43(39.44 \%)$ strains of S. aureus were isolated and among that one third were MRSA. Antibiotic resistant pattern of MRSA strains were found to be highly variable. Resistance to ampicillin, cefuroxime, ciprofloxacin, netilmicin and amikacin were found to be $76.9 \%$, $76.9 \%, 53.8 \%, 23 \%$ and $23 \%$ respectively. More than one tenth of the nursing staff were at risk of transmitting the infection and therefore standard infection control precautions should be followed to minimise carriage and transmission.
\end{abstract}

Ceylon Medical Journal 2014; 59: 63-65

\section{Introduction}

Methicillin-resistant Staphylococcus aureus (MRSA) is a specific strain of S. aureus that has developed antibiotic resistance to all $\beta$-lactams [1]. MRSA causes a variety of diseases ranging from mild, superficial dermatological infections to severe and potentially fatal systemic infections [2]. MRSA asymptomatically colonise the nasal cavity, axillae and groin region of patients and transient carriage in hands may occur due to the poor hand hygiene. Health care workers are the major source of MRSA in the hospital environment. Hand washing is the most important method in the prevention of transmission of MRSA [4].

Previous studies in Sri Lanka have shown that approximately $12 \%-47 \%$ of patients were positive for MRSA $[1,2]$. Other studies have proposed that healthcare workers may act as carriers for MRSA transmission [5]. Therefore we set out to determine MRSA carriage in hands among health care workers in the Teaching Hospital, Jaffna, Sri Lanka as identifying transient carriage of hands is important in preventing spread by health care workers.

\section{Methods}

The study was approved by the Ethical Review Committee of the Faculty of Medicine, University of Jaffna, Sri Lanka. This descriptive study was carried out to identify the occurrence of MRSA carriage among the nursing staff in different units of Jaffna Teaching Hospital. The study 